\title{
Vorwort zur 5. Auflage
}

Überraschend schnell war die vierte Auflage dieses Buches vergriffen. Die Neuauflage wurde von uns auf den neuesten Stand von Ende 1969 gebracht und die zahlreichen, mannigfaltigen Publikationen auf dem engeren Wassergebiet bis 1969 beriucksichtigt. Einige der freundlicherweise zur Einsicht übersandten Manuskripte, die 1970 veröffentlicht werden, konnten ebenfalls noch berücksichtigt werden. Die fünfte Auflage soll daher für einige Zeit etwas Endgültiges darstellen.

Nach wie vor ist das Buch in erster Linie dem Trinkwasser gewidmet; da Trinkwasser, Betriebswasser, Badewasser, Flußwasser und Abwasser eng miteinander verflochten sind und ineinander übergehen, werden auch diese Gebiete mehr oder weniger eingehend behandelt. So spielt z. B. eine Polyphosphatbehandlung des Trinkwassers eine große Rolle bei der Nutzung desselben als Schwimmbadwasser (durch Hervorrufen unerwünschter Algenentwicklungen) und über das Abwasser in den Gewässern als Algennährstoff.

Die Filtertechnik wurde in Verbindung mit der Wasserchemie neu behandelt (z. B. Mehrschichtfiltration).

Der Teil Abwasser ist weiterhin sehr kurz gehalten, ebenso wie der biologische Beitrag von Herrn Direktor Dr. Lüdemann. Auf diesen Gebieten sind kürzlich umfangreiche Spezialwerke erschienen. Bei Mineralwasser und Heilwasser ist dies noch nicht der Fall; deshalb wird dieser Abschnitt in der 5 . Auflage mit geringen Änderungen beibehalten, um die Grenzen Trinkwasser - Mineralwasser - Heilwasser beurteilen zu helfen z. B. bei Neuerschließungen.

Völlig neu bearbeitet wurde der Abschnitt über Schwimmbadwasser im chemischen und bakteriologischen Teil wegen des großen Fortschritts auf diesem Gebiet sowie das Kapitel Chlor-Nachweis.

Das Buch ist aus der Praxis für die Praxis leicht verständlich geschrieben, um allen Wasserfachleuten, Hygienikern, Technikern, Biologen, Chemikern u. a. die so notwendige Zusammenarbeit zu erleichtern. Auf theore tische Erörterungen und chemische Formeln wurde deshalb weiterhin verzichtet.

Trotz der Verbesserung der apparativen Ausrüstung vieler Wasserlaboratorien konnte m. E. auf einfache Methoden wie z. B. die der visuellen Kolorimetrie nicht verzichtet werden. Bei Feldarbeiten und Expeditionen sowie bei Sonderbestimmungen ist man auf diese angewiesen. G. Kortüm räumt der visuellen Kolorimetrie in seinem bekannten Werk auch jetzt noch gewisse Vorteile vor anderen Verfahren ein. Die Analysenvorschriften berücksichtigen im übrigen die bisherigen Einheitsverfahren der Fachgruppe; auf diesen sind ja schließlich auch die Beurteilungsgrundsätze aufgebaut.

Die im Text angeführten Literatur-Stellen sind in erster Linie als Beleg für die mitgeteilten neuen Erkenntnisse und Forderungen der Wasserchemie gedacht; der Kürze halber 
ist bei diesen daher auf die oft langen Titel der Zeitschriften-Veröffentlichungen verzichtet worden.

Die Wasserchemie gewinnt infolge der großen Probleme der Wasserversorgung z. B. der der Fernwasserversorgung aus Talsperren u. a. Oberflächengewässern immer größere Bedeutung. Diese Probleme können nur durch Zusammenarbeit aller Disziplinen der Wasserwirtschaft vorangetrieben und gelöst werden. Hierzu möchte das Buch einen Beitrag liefern.

Herrn Direktor Dr. Lüdemann, Berlin, und Herrn Dr. med. Peter danke ich für die weitere Mitarbeit.

Herrn Prof. Dr. Hässelbarth und Herrn Dr. Grohmann vom Bundesgesundheitsamt Berlin (Wa Bo Lu) danke ich verbindlichst für die eingehende Durchsicht der Kapitel Aggressivität von Wässern und die Ausrichtung auf die neuesten Erkenntnisse vom Jahr 1970.

Hameln, Frühjahr 1970

Dr. Karl Höll 
